\title{
INFLUENCIA DEL CONTROL ALTITUDINAL EN LA DISTRIBUCIÓN DE UNIDADES MORFODINÁMICAS DEGRADATIVAS Y AGRADATIVAS EN ÁREAS DE MONTAÑA: EL CIRCO DEL TAILLON (VALLE DE GAVARNIE, PIRINEO CENTRAL FRANCÉS)
}

\author{
Javier CHUECA CÍA y Asunción JULIÁN ANDRÉS \\ Departamento de Geografía y Ordenación del Territorio \\ Universidad de Zaragoza
}

\begin{abstract}
Resumen: En este trabajo se comprueba - de una forma cuantitativa- la existencia de un control altitudinal en el reparto espacial de las unidades morfodinámicas identificadas en el circo glaciar del Taillon. La metodología utilizada, que parte de la cartografía y posterior planimetrado de las distintas subunidades degradativas y agradativas definidas, permite establecer el grado de madurez del sector montañoso estudiado con notable precisión.
\end{abstract}

Palabras clave: Control altitudinal, unidades morfodinámicas, Pirineo central francés.

\begin{abstract}
In this work the existence of an altitudinal control in the spatial

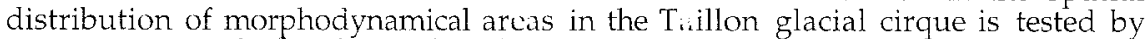
quantitative methods. The methodology adopted starts with the cartography and subsequent planimetry of the different agradative and degradative subunities defined, and allows us to stablish acurately the degree of maturity of the mountainous sector studied.
\end{abstract}

Key words: Altitudinal control, morphodynamical areas, French central Pyrenees.

\section{INTRODUCCIÓN}

El área estudiada en este trabajo se emplaza en la vertiente francesa de los Pirineos centrales, dentro del sector oriental de la cara norte del macizo de Monte Perdido e inmerso en la pequeña Gavé des Tourettes, afluente del principal eje fluvial rector de la zona, el Valle de Gavarnie (Fig. 1). Abarca lo que hemos denominado globalmente circo glaciar del Taillón, extensión de en torno a los $2 \mathrm{~km}^{2}$ drenada por el arroyo que fluye desde el glaciar del Taillon y que se alarga en dirección norte desde el pie de las cumbres cimeras, con elevaciones que llegan a superar o rondan los $3.000 \mathrm{~m}$ 
(Taillon, $3.144 \mathrm{~m}$; Pointe Bazillac, $2.975 \mathrm{~m}$ ), hasta llegar a la confluencia de este barranco con la ya mencionada Gavé des Tourettes, a unos 1.800-1.900 m de altura.

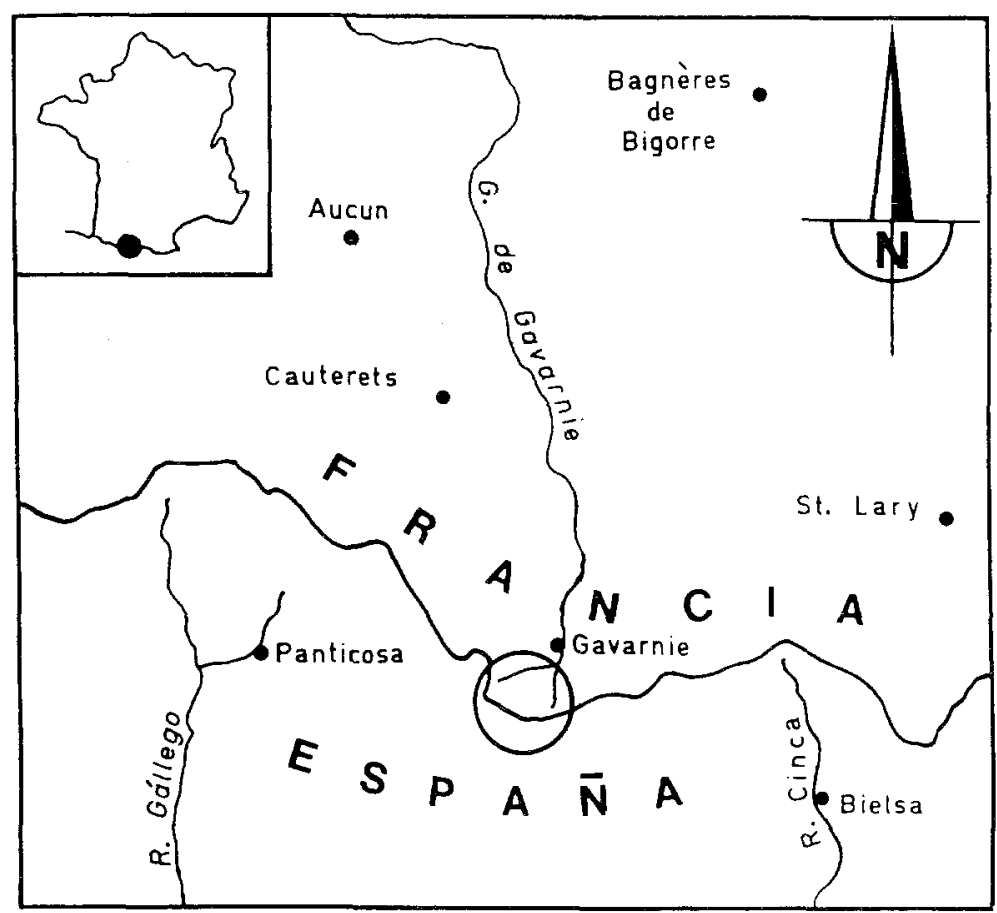

Figura 1.- Localización del área de estudio.

La zona elegida exhibe las características típicas de un espacio de alta montaña glaciado pirenaico: al aspecto austero, abrupto y verticalizado que da el roquedo desnudo en el sector somital, se une el hielo glaciar y las grandes acumulaciones caóticas de bloques (acumulaciones de gravedad, depósitos morrénicos) del tramo intermedio y los pastizales alpinos y pseudoalpinos que dominan las vertientes en su porción final. Estas diferencias, que paisajísticamente son tan notables, hicieron fácil el planteamiento de este trabajo: comprobar -de una forma cuantitativa- la existencia de un control altitudinal en el reparto espacial de las unidades morfodinámicas degradativas y agradativas identificadas en la zona de análisis. El estudio adquiere mayor significación si se tiene en cuenta el citado carácter típico -en términos morfodinámicos- del circo del Taillon, rasgo éste que proporciona una representatividad general a los resultados obtenidos y los hace extensibles, si no 
obviamente a la totalidad de circos pirenaicos (que pueden encontrarse en etapas del proceso de deglaciación bien diferentes), sí cuando menos a los que aún albergan en su interior modestos aparatos glaciares.

\section{ZONA DE ESTUDIO}

Acabamos de apuntar los rasgos topográficos básicos que caracterizan a la zona de estudio; desde un punto de vista geológico, el circo glaciar del Taillon se ubica prácticamente en el límite entre la Zona Axial pirenaica, en donde aparece el basamento correspondiente a los materiales del ciclo hercínico (que afloran en la margen izquierda de la Gavé des Tourettes), y la zona Sur-pirenaica, constituida por las series sedimentadas y deformadas durante el ciclo alpino que forman parte de Ia unidad estructural del Manto de Gavarnie (aflorantes en la margen derecha de 1 a Gavé des Tourettes) (RÍOS et al., 1989). Los materiales que arman el circo estudiado son básicamente de naturaleza calcárea, calcoarenosa o dolomítica, y abarcan desde el Cretácico Superior (Cenomaniense-Coniaciense) de las cotas altitudinales más bajas, hasta el Terciario Inferior (Paleoceno) de los sectores cimeros; destacan por su extensión espacial los afloramientos de calizas arenosas y areniscas de cemento calcáreo de la Formación Tozal (Campaniense-Maastrichtiense), que generan los escarpes más abruptos de la zona de análisis y se localizan aproximadamente entre los 2.300 y $2.900 \mathrm{~m}$ de altura s.n.m. En todo el circo del Taillon es general el buzamiento de las capas hacia el S (con valores suavizados conforme ascendemos en altura), generado por un deslizamiento capa a capa mediante fallas inversas que provoca la repetición de las series por el apilamiento de escamas. Esta estructura condiciona el aspecto morfológico general de todo el macizo, que exhibe una marcada disimetría entre las laderas norte (francesas) y sur (españolas): en las vertientes norte -como la que nos ocupa- los relieves son más abruptos, ya que la erosión aprovecha para actuar la esquistosidad de presión-disolución existente con pronunciados buzamientos $\mathrm{N}$; las vertientes sur están ocupadas preferentemente por las calizas del Terciario basal estructuradas según pliegues en cascada que dan lugar a las mesetas estructurales, horizontales o alabeadas, de relieve maduro, tan características en todo el macizo de Ordesa y Monte Perdido.

Climáticamente estamos ante un espacio de alta montaña que, como es lógico, se caracteriza por una marcada crudeza ambiental; no existe en la zona de estudio ni en su proximidad más inmediata ningún observatorio meteorológico, pero podemos tomar como referencia -siempre teniendo en cuenta que están basadas en estaciones ubicadas en la vertiente española del macizo (Góriz, Linás de Broto, Fanlo, Broto, etc.)- los valores aportados por GARCÍA-RUIZ y MARTÍ BONO (1993) en su completo trabajo sobre el Parque Nacional de Ordesa y Monte Perdido. Estos autores hablan para la 
zona de unas influencias atlánticas ya muy debilitadas que se traducen en la recepción de un mayor volumen de precipitaciones en otoño y primavera y en un claro descenso en el invierno y en época estival; precipitaciones que son en cualquier caso importantes a 1 tratarse de un área de montaña (podemos citar los ejemplos de Linás de Broto, a unos $1.330 \mathrm{~m}$ de altura, donde se registran como media anual $1.635 \mathrm{~mm}$ de precipitación; Fanlo, a $1.320 \mathrm{~m}$, con $1.621 \mathrm{~mm}$; o Broto, a $1.005 \mathrm{~m}$, con $\mathrm{un}$ registro de $1.323 \mathrm{~mm}$ ). Lógicamente, estos valores aún deben ser mayores en cotas superiores y dentro de vertientes norteñas como la analizada en nuestro trabajo, sometidas a um influjo oceánico mucho mayor; por otro lado, durante buena parte del invierno, aunque no excesivas, las precipitaciones registradas van a ser predominantemente nivosas, con 10 que ello supone de cara a alimentar determinados procesos dinámicos de tipo geomorfológico. Térmicamente, las temperaturas se hallan también muy relacionadas con la altura, y por tanto van a alcanzar valores muy bajos: así, en Góriz, a $2.220 \mathrm{~m}$ de altitud, la temperatura media invernal es inferior a los $0^{\circ} \mathrm{C}$, en Fanlo, es de $2^{\circ} \mathrm{C}$ y en Broto de $3,4^{\circ} \mathrm{C}$; de acuerdo con los cálculos de GARCÍA-RUIZ et al. (1985), la isoterma de $0^{\circ} \mathrm{C}$ durante la estación fría (noviembre-abril) en la vertiente española del macizo se sitúa en cotas bajas, a unos 1.600-1.670 m, hecho que repercute sin duda en $1 \mathrm{a}$ potenciación estacional de los procesos ligados a la nivación en la zona estudiada. Una mínima extrapolación en altura de los valores térmicos citados nos permite apreciar que a cotas por encima de los 2.400-2.500 m, las temperaturas entrarian ya en el dominio pirenaico de los territorios climáticos extremadamente fríos a muy fríos (característicos de los pisos morfodinámicos nival y subnival; CHUECA, 1993), con claras repercusiones en la aparición de procesos geomorfológicos morfodinámicos de degradación y agradación de tipo periglaciar y, más marginalmente, glaciar.

Bajo estos condicionantes climáticos, las únicas comunidades vegetales que presentan cierta extensión y continuidad en la zona estudiada son los pastizales pseudoalpinos y alpinos. En el primero de los casos -en las franjas altitudinales más bajas, por debajo aproximadamente de los 2.100-2.200- estamos hablando de las tipicas tascas pirenaicas de gramíneas, comunidades relativamente complejas y ricas en especies, aunque emplazadas en ámbitos potencialmente forestales [los ejemplares aislados de pino negro (Pinus uncinata), por poner un ejemplo, pueden ascender en sectores próximos hasta cotas cercanas a los $2.500 \mathrm{~m}$ ); en la zona -deforestada hace ya largos años para proporcionar pastos al ganado- únicamente se conservan algunos ejemplares aislados de porte arbustivo que salpican los pastizales ocasionalmente: Rhododendron ferrugineum, Vaccinium myrtillus, V. uliginosum, etc.]. Por encima de los 2.100-2.200 m, las manchas aisladas de comunidades herbáceas alpinas -pastos de altura, abiertos, y menos ricos florísticamente- van alternándose con el roquedo desnudo, creciendo la extensión de éste conforme nos desplazamos en altura y los suelos se hacen cada vez menos potentes y más inmaduros; este tipo de pastos sí representan la vegetación natural del área de análisis, y están formados en las zonas más favorables por macollas más o menos densas de gramíneas (Festuca sp., Carex sp., etc.), 
acompañadas en enclaves particulares por las especialistas de distintas comunidades: rupícolas, de neveros, de tramos turbosos, etc.

\section{MÉTODO DE TRABAJO Y RESULTADOS}

\section{El método de trabajo: definición de unidades morfodinámicas}

Dentro de la zona de estudio cuyos rasgos generales acabamos de exponer, y atendiendo ya a un punto de vista morfodinámico, nos encontramos con la existencia de dos grandes tipos de unidades: 1) las unidades morfodinámicas degradativas, en las que lo que predomina es la destrucción y erosión de las morfologías preexistentes; y 2) las unidades morfodinámicas agradativas, con predominio de la acumulación de materiales sobre morfologías preexistentes.

Cualquier zona de montaña exhibe este tipo de organización, pero el número de procesos identificables en cada sector -y la intensidad que alcanza su actuación- es, lógicamente, muy variable, y depende de factores tales como la energía de relieve, que va a condicionar que el flujo de materiales sea más o menos rápido e intenso; la latitud y altura en la que la zona se ubica y su orientación, que incidirán respectivamente en la acentuación de la crudeza climática a nivel general y de detalle; o la presencia de una más o menos densa cubierta vegetal y de las características fisionómicas de ésta (es bien conocida, por ejemplo, la atenuación del dinamismo de muchos procesos en sectores con cubierta forestal densa). En el circo del Taillon, nos encontramos con un espacio en el que a una energía de relieve considerable (pasamos de los $3.144 \mathrm{~m}$ de la cumbre del Taillón, a los $1.830 \mathrm{~m}$ en la Gavé des Tourettes, en apenas un par de kilómetros), se une la orientación en umbría y la existencia de una cobertera vegetal muy escasa, herbácea y localizada únicamente de forma masiva en la parte más baja del circo: una zona, por tanto, proclive a mostrar un dinamismo geomorfológico importante (Foto 1).

Las subunidades morfodinámicas identificadas en la zona incluyen siete categorías básicas, tres de tipo degradativo y cuatro agradativo. Dentro del tipo degradativo, se han reconocido las siguientes: 1) escarpes y vertientes rocosas: dominio de procesos criogénicos intensos, son los sectores donde predomina la roca desnuda afloramientos de los materiales fundamentalmente carbonatados (calizas, calcarenitas, dolomías, etc.) del Cretácico Superior/Terciario Inferior que ya hemos apuntado previamente-, con pendientes elevadas en general (paredes verticales o incluso extraplomadas, a veces) y frecuentemente emplazados en las zonas convexas cimeras de divisoria y en su continuación inmediata hacia el fondo del circo del Taillon y de los circos adyacentes; 2) canales de incisión: son relativamente numerosos en toda el área cartografiada y abundan en los tramos de escarpes y vertientes rocosas 
surcados por zonas de debilidad con fracturación, diaclasado o esquistosidad (fundamentalmente sobre las calizas arenosas y areniscas de la Formación Tozal); la gran mayoría sólo actúa episódicamente como sectores de canalización: de agua y/o flujos de derrubios tras períodos de lluvia intensos, de aludes o avalanchas, o de desprendimientos rocosos; y 3) aparatos glaciares: el pequeño glaciar del Taillon, típica morfología de glaciar pirenaico en circo, muestra todavía trazas -a pesar de su evidente degradación- de una cierta movilidad, y por tanto de un poder erosivo, aspecto éste que permite incluirlo plenamente dentro de las subunidades morfodinámicas degradativas. Por su parte, encuadradas en el tipo agradativo, aparecen: 4) morrenas y derrubios de nivación: generados como es lógico por la dinámica glaciar y nival, se extienden en la zona de estudio desde los $2.500 \mathrm{~m}$ hasta los $1.900 \mathrm{~m}$ de altura, y corresponden a tres fases de deglaciación holocenas [etapa Neoglaciar para los arcos externos -datada estimativamente por analogía con las morrenas identificadas por GELLATLY et al. (1992) en el vecino circo de Troumouse-; etapa de la Pequeña Edad del Hielo para los arcos intermedios; y etapa actual o subactual para los mucho más pequeños arcos internos que se encuentran prácticamente en contacto con la masa de hielo actual]; 5) cubiertas de derrubios: normalmente ubicadas al pie de los grandes escarpes y vertientes rocosas ya mencionados, engloban tanto a los típicos canchales o gleras con morfología en mantos irregulares (alimentados por procesos gravitacionales, de deslizamiento y salto de clastos), como a canchales en conos y a conos de aludes (muy escasos ambos en estado puro) y a conos mixtos (más frecuentes en la zona y generados fundamentalmente por la acción alternante de episodios de aludes, de episodios torrenciales con flujos de derrubios, y de los mencionados procesos de gravedad); 6) vertientes de geli-solifluxión: características en aquellos lugares con cobertera herbácea -ya en cotas relativamente bajas del circo del Taillon- donde comienzan a aparecer suelos de una cierta potencia y plasticidad, se traducen sobre el terreno en las típicas morfologías abombadas, en lóbulos y mantos, generadas por los movimientos en masa de tipo lento (conviene recordar de nuevo que nos encontramos en una zona de tascas de carácter claramente pseudoalpino, en un ámbito forestado -de ahí la relativa potencia y riqueza de estos suelos- hasta época no muy lejana); y 7) fondos de valle: escasísimos en la zona estudiada, son tramos de acumulación de coluviones con topografía en terrazas aplanadas o subaplanadas dominados, en nuestro caso, por una dinámica fluvial torrencial episódica.

El método de trabajo que adoptamos incluía efectuar, en primer lugar, una cartografía geomorfológica a escala detallada (1:15.000, aproximadamente) de la zona de estudio, cartografía en la que estuvieran contemplados los componentes de las unidades y subunidades morfodinámicas que, posteriormente $-\mathrm{y}$ tras la pertinente comprobación sobre el terreno- serían objeto de medición y planimetría (Fig. 2). 


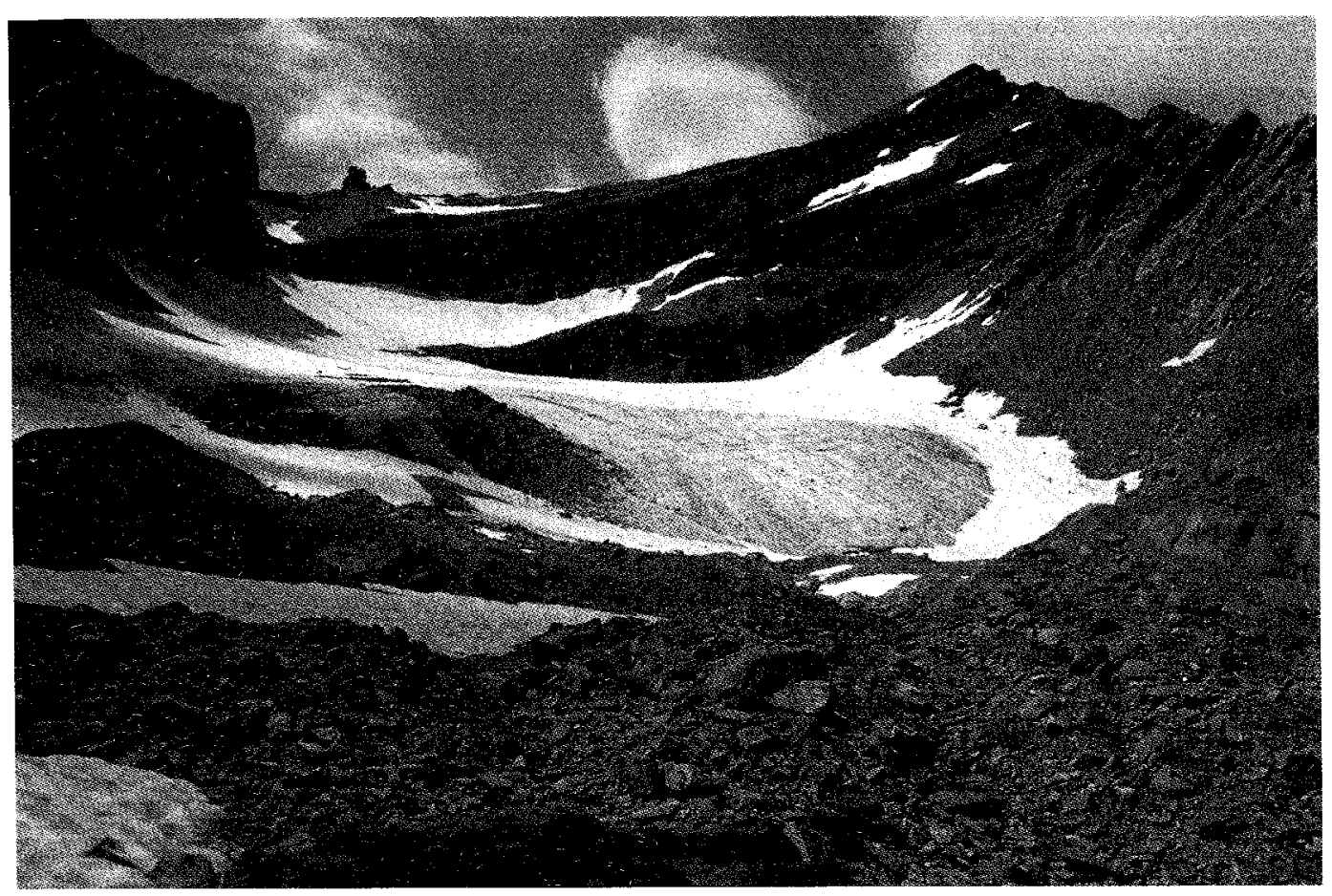

Fotografía 1.- Panorámica del sector superior del circo glaciar del Taillon desde las proximidades del Col des Sarradets $(2.589 \mathrm{~m})$.

La cartografía se realizó en gabinete mediante el empleo de un planímetro digital y por los procedimientos usuales (lecturas múltiples para cada subunidad y unidad y toma de valores medios), intentando minimizar el grado de error al efectuar una única persona todas las tareas de lectura; las superficies se planimetraron por estratos altitudinales en siete categorías, abarcando en general cada intervalo unos $200 \mathrm{~m}$ de altitud: 1) alturas $<1.900 \mathrm{~m}$; 2) $1.900-2.100 \mathrm{~m}$; 3) $2.100-2.300 \mathrm{~m}$; 4) $2.300-2.500$ $\mathrm{m}$; 5) 2.500-2.700 m; 6) 2.700-2.900 m y 7) >2.900 m. Como único antececedente previo llevado a cabo en un sector pirenaico contábamos con los estudios realizados por CHUECA (1993 a, b) para la Alta Ribagorza aragonesa, estudios sin embargo de tipo cualitativo ya que, aunque se exponían en ellos los aspectos generales del morfodinamismo de la zona, no se llegaba a realizar una cartografía específica de detalle de las unidades agradativas y degradativas identificadas ni su planimetría, dada la menor escala de trabajo adoptada. 


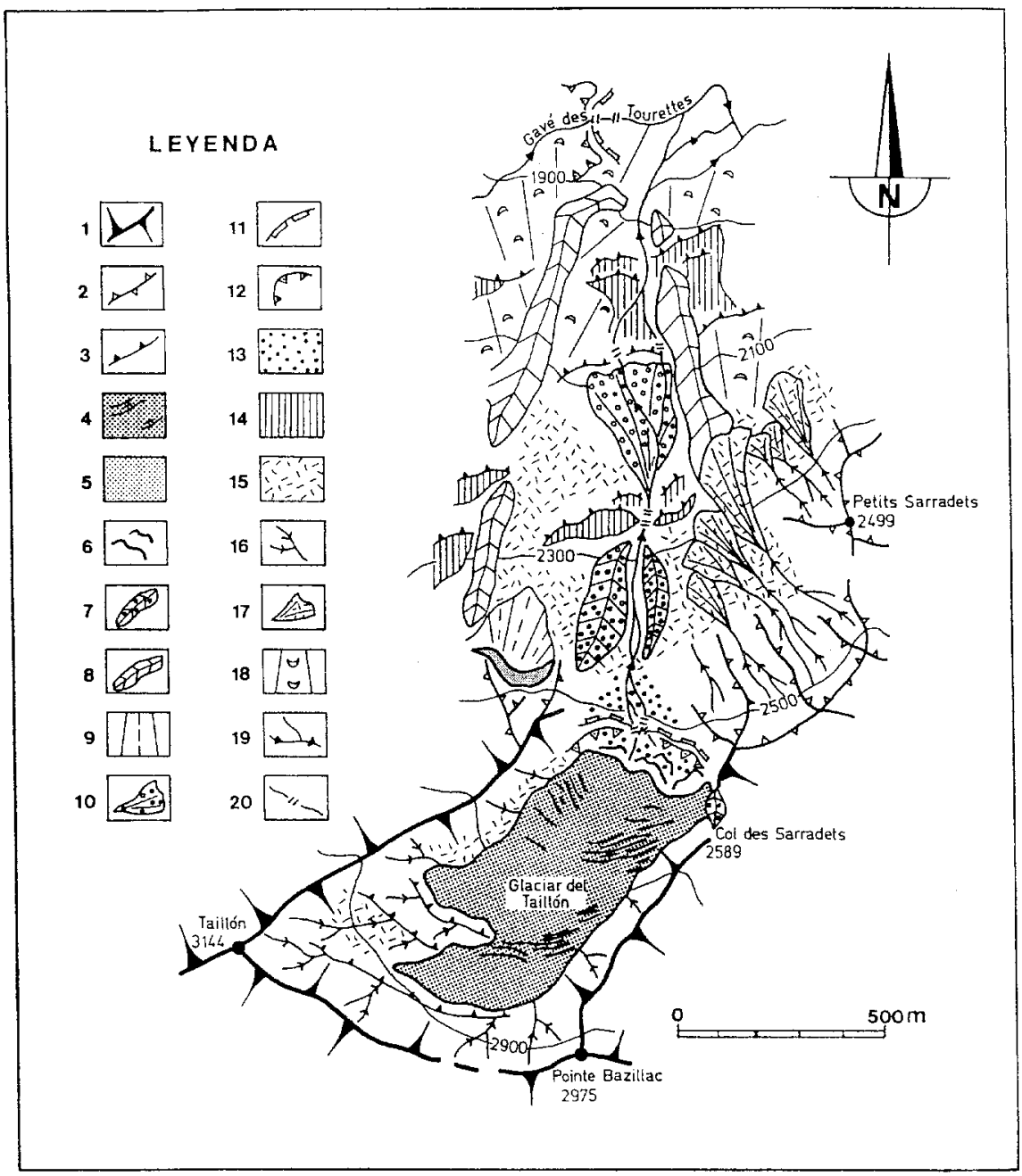

Figura 2.- Cartografía geomorfológica del área de estudio. Leyenda: 1) Límites de circos glaciares; 2) Cordales de divisoria de cuencas; 3) Escarpes menores de origen estructural; 4) Glaciares; 5) Neveros semipermanentes; 6) Depósitos morrénicos actuales y subactuales; 7) Depósitos morrénicos de la Pequeña Edad del Hielo; 8) Otros depósitos morrénicos; 9) Derrubios de nivación; 10) Conos proglaciares; 11) Umbrales de origen glaciar; 12) Cubetas de sobreexcavación glaciar; 13) Rocas pulidas y aborregadas; 14) Áreas karstificadas; 15) Cubiertas de derrubios típicas (canchales); 16) Canales de aludes; 17) Conos de aludes/Conos mixtos; 18) Áreas con procesos de geli-solifluxión; 19) Red fluvial; 20) Cambios bruscos de pendiente en la red fluvial. 


\section{La distribución altitudinal de unidades morfodinámicas}

Los resultados obtenidos tras efectuar estas tareas aparecen plasmados en 1 a Tabla I, en la que se recogen los valores totales y subtotales de extensión superficial por estratos altitudinales de las unidades y subunidades degradativas y agradativas identificadas en el área de análisis, y ya mencionadas en el apartado anterior. De su examen se deducen varias consecuencias:

1) En primer lugar, se constata la existencia de una práctica similitud entre los valores de extensión superficial de las unidades degradativas $\left(0,955 \mathrm{~km}^{2}, 50,6 \%\right)$ frente a las agradativas $\left(0,931 \mathrm{~km}^{2}, 49,4 \%\right)$, rasgo indicativo de la presencia de $\mathbf{m}$ notable equilibrio global en la organización morfodinámica del circo glaciar estudiado en su actual momento evolutivo (a este respecto, sería interesante realizar análisis similares en circos glaciares en fases evolutivas diferentes, de cara a confirmar la existencia de unas más que probables diferencias en los valores superficiales degradación-agradación).

2) En segundo lugar, se hace evidente la clara preponderancia espacial dentro del conjunto degradativo de la subunidad "escarpes y vertientes rocosas", con valores muy por encima de los de las otras dos subunidades identificadas (escarpes y vertientes rocosas: $0,585 \mathrm{~km}^{2}, 61,2 \%$; canales de incisión: $0,152 \mathrm{~km}^{2}, 15,9 \%$; aparatos glaciares: 0,218 $\mathrm{km}^{2}, 22,9 \%$ ); por el contrario, dentro del conjunto agradativo, las cuatro subunidades identificadas -con la matizada excepción de los fondos de valle- registran valores de extensión superficial muy semejantes (morrenas y derrubios de nivación: $0,277 \mathrm{~km}^{2}$, $29,8 \%$; cubiertas de derrubios: $0,289 \mathrm{~km}^{2}, 31 \%$; vertientes de geli-solifluxión: $0,247 \mathrm{~km}^{2}$, $26,6 \%$; fondos de valle: $0,118 \mathrm{~km}^{2}, 12,6 \%$ ).

La preponderancia espacial tan marcada de una subunidad degradativa concreta responde en gran medida a la abrupta naturaleza topográfica de la zona (típica de un sector montañoso joven en términos geológicos), condicionada primeramente por los factores estructurales y litológicos ya apuntados, y posteriormente reafirmada - a lo largo del Cuaternario- por la actuación en el área de estudio de un glaciarismo de notable importancia.

3) En tercer lugar, a la vista de los valores totales y subtotales registrados, se hace también evidente la presencia de un marcado cambio en los valores de extensión superficial del binomio agradación-degradación a partir de una determinada altura: en efecto, este cambio se observa a partir del estrato altitudinal de $\operatorname{los} 2.300-2.500 \mathrm{~m}$, franja en la que hay un casi total equilibrio en la extensión espacial de los procesos morfodinámicos degradativos frente a los agradativos $(2.300-2.500 \mathrm{~m}$ : unidad degradativa: $0,190 \mathrm{~km}^{2}, 48 \%$ / unidad agradativa: $0,206 \mathrm{~km}^{2}, 52 \%$ ). Hasta esa altura, en los distintos estratos altitudinales trabajados predominaban las superficies de 
agradación sobre las de degradación (<1900 m: unidad degradativa: 0,026 km², 12,2\% / unidad agradativa: $0,187 \mathrm{~km}^{2}, 87,8 \% ; 1.900-2.100 \mathrm{~m}$ : unidad degradativa: $0,103 \mathrm{~km}^{2}$, $34,3 \%$ / unidad agradativa: $0,197 \mathrm{~km}^{2}, 65,7 \% ; 2.100-2.300 \mathrm{~m}$ : unidad degradativa: $0,078 \mathrm{~km}^{2}, 22,1 \%$ / unidad agradativa: $0,274 \mathrm{~km}^{2}, 77,9 \%$ ). Por su parte, a partir de los 2.300-2.500 $\mathrm{m}$ pasan a predominar espacialmente los procesos morfodinámicos degradativos frente a los agradativos (2.500-2.700 m: unidad degradativa: $0,211 \mathrm{~km}^{2}$, $84,7 \%$ / unidad agradativa: $0,038 \mathrm{~km}^{2}, 15,3 \% ; 2.700-2.900 \mathrm{~m}$ : unidad degradativa: $0,240 \mathrm{~km}^{2}, 93 \%$ / unidad agradativa: $0,018 \mathrm{~km}^{2}, 7 \%$; $>2.900 \mathrm{~m}$ : unidad degradativa: $0,107 \mathrm{~km}^{2}, 90,6 \%$ / unidad agradativa: $\left.0,011 \mathrm{~km}^{2}, 9,4 \%\right)$.

Este brusco cambio de tendencia marca claramente los dominios espaciales respectivos de una montaña inestable, de dinámica erosiva intensa (cotas altas), y los de una montaña estabilizada o pseudoestabilizada, con procesos morfodinámicos de acumulación (cotas bajas).

4) En cuarto lugar, analizando la evolución de los valores de extensión superficial obtenidos para cada subunidad por estratos altitudinales, se observan igualmente diferencias significativas (los valores absolutos, aunque importantes per se, se ven complementados por los porcentuales, que permiten hacerse con una idea más clara del peso espacial respectivo de cada subunidad dentro del total de cada estrato altitudinal considerado; por eso son los utilizados aquí): a) la extensión espacial de la subunidad escarpes y vertientes rocosas registra una clara tendencia ascendente desde el valor mínimo observado en las cotas inferiores a $1.900 \mathrm{~m}\left(0,018 \mathrm{~km}^{2}, 8,4 \%\right.$ del total de subunidades del estrato), hasta el máximo, localizado por encima de los $2.900 \mathrm{~m}(0,088$ $\left.\mathrm{km}^{2}, 74,5 \%\right)$; b) similar pauta exhibe la subunidad canales de incisión, que registra un mínimo en las cotas inferiores a $1.900 \mathrm{~m}\left(0,008 \mathrm{~km}^{2}, 3,7 \%\right)$, y un máximo por encima de $\operatorname{los} 2.900 \mathrm{~m}\left(0,019 \mathrm{~km}^{2}, 16,1 \%\right)$; c) la subunidad aparatos glaciares únicamente aparece representada en dos estratos altitudinales: el valor máximo se observa en la franja $2.700-2.900 \mathrm{~m}\left(0,113 \mathrm{~km}^{2}, 43,7 \%\right)$; d) la subunidad morrenas y derrubios de nivación se localiza entre la base del circo y el estrato de los 2.500-2.700 m; el valor máximo se da entre los 2.100-2.300 $\mathrm{m}\left(0,094 \mathrm{~km}^{2}, 26,7 \%\right)$; e) las cubiertas de derrubios están ausentes en los dos estratos inferiores y su valor máximo se registra igualmente entre los 2.100$2.300 \mathrm{~m}\left(0,138 \mathrm{~km}^{2}, 39,2 \%\right)$; ) la subunidad vertientes de geli-solifluxión aparece tan sólo a lo largo de las tres primeras franjas altitudinales, y su máximo espacial se constata en el estrato $1.900-2.100 \mathrm{~m}\left(0,135 \mathrm{~km}^{2}, 45 \%\right)$; g) por último, la subunidad fondos de valle únicamente se observa en la parte más baja del circo, en las dos franjas altitudinales inferiores: su máximo se da en el estrato de alturas inferiores a $1051.900 \mathrm{~m}(0,113 \mathrm{~km}$, $53 \%$ ). 

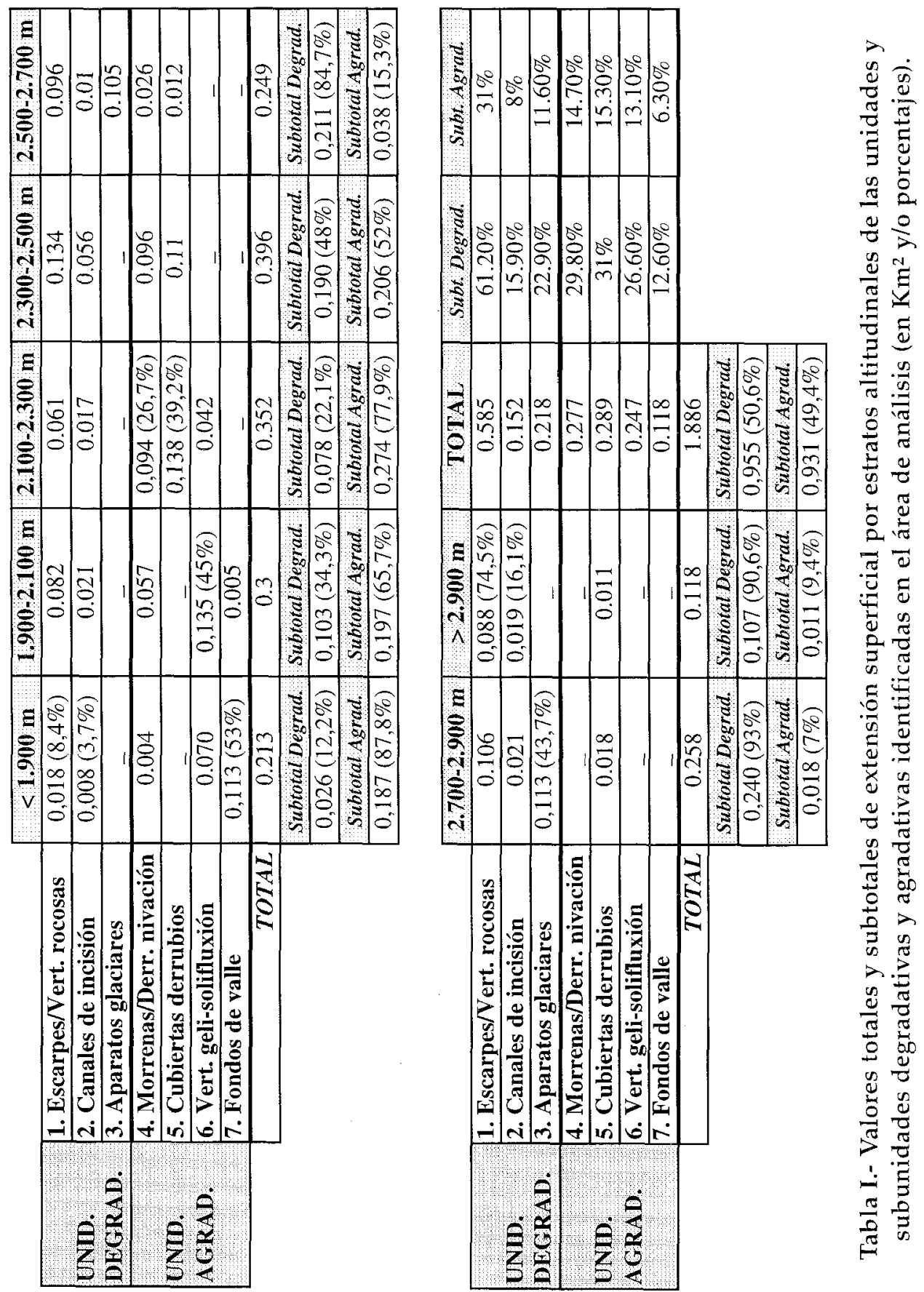
Las tendencias particulares observadas permiten matizar los valores que hemos comentado en el apartado anterior: la brusca transición entre el predominio de unidades agradativas y el de degradativas se hace aquí también evidente pero, como puede observarse, las dos únicas subunidades que tienen representación espacial a lo largo de todas las franjas altitudinales -incluso, y queremos resaltarlo, en las más bajas- son las degradativas escarpes y vertientes rocosas y canales de incisión. Este hecho -la extensión/invasión de procesos morfodinámicos degradativos en un marco predominantemente agradativo- va a favorecer también la presencia de una inestabilidad potencial elevada, aunque de carácter puntual, en los tramos inferiores del circo.

\section{CONCLUSIONES}

En conjunto, y a la vista de los datos manejados, parece claro que podemos hablar en la zona analizada de la existencia actual de un cierto equilibrio global en el balance agradación-degradación (con un ligero predominio en la extensión espacial de los procesos morfodinámicos degradativos sobre los agradativos). Sin embargo, como ya hemos comentado, el examen detallado por pisos altitudinales de la extensión de las dos unidades estudiadas, nos permite constatar sin ninguna duda que nos encontramos ante un sector montañoso en gran medida inestable. Inestabilidad que se hace mucho más evidente en cotas altitudinales elevadas, por encima de los 2.300 $2.500 \mathrm{~m}$, sectores donde la intensidad en los procesos degradativos de transferencia de materia y energía alcanza su máxima expresión. Por debajo de esos 2.300-2.500 m de altura nos encontraríamos en un ámbito más maduro, con una marcada tendencia hacia la estabilización o la pseudoestabilización controlada por el predominio espacial de los mecanismos morfodinámicos agradativos, tendencia que, sin embargo y puntualmente, puede verse afectada por la actuación de determinados procesos de degradación.

Parece claro, en cualquier caso, que podemos basarnos en el estudio de este binomio degradación-agradación (o, utilizando otros términos, erosión-acumulación) para caracterizar de una forma relativamente sencilla sectores de montaña desde el punto de vista de su dinamismo geomorfológico, puesto que los resultados obtenidos permiten hacernos con una idea muy precisa de cuales son los procesos dominantes en cada estrato altitudinal considerado $y$, consecuentemente, del grado de madurez general del conjunto. El procedimiento descrito aquí podría ser además muy útil para efectuar una cartografía tipificada de detalle de zonas de montaña -y quizá también de otros ámbitos- por lo que, pensamos, merece ser experimentado en el futuro en otras áreas. 


\section{BIBLIOGRAFÍA}

CHUECA, J. (1993 a): Geomorfología de la Alta Ribagorza aragonesa. Asociación Guayente, 250 p. Benasque (Huesca).

CHUECA, J. (1993 b): "Definición, funcionamiento y estructuración de geocomplejos morfodinámicos en la Alta Ribagorza aragonesa". Geographicalia. 30, 69-86, Zaragoza.

GARCÍA-RUIZ, J.M. y MARTÍ BONO, C. (1993): Mapa geomorfológico del Parque Nacional de Ordesa y Monte Perdido. Instituto Pirenaico de Ecología, 117 p. Zaragoza.

GARCIA-RUIZ, J.M., PUIGDEFÁBREGAS, J. y CREUS, J. (1985): Los recursos hidricos superficiales del Alto Aragón. Instituto de Estudios Altoaragoneses, 224 p. Huesca.

GELLATLY, A.F., GROVE, J.M. y SWITSUR, V.R. (1992): Mid-Holocene glacial activity in the Pyrenees. The Holocene. 2-3, 266-270, London.

RIOS, L.M., GALERA, J.M., BARETTINO, D. y BARNOLAS, A. (1989): Mapa Geológico de España, escala 1:50.000, Hoja 146, Bujaruelo. Instituto Tecnológico Geominero de España, 64 p. Madrid. 